\title{
Teste de Condutividade Elétrica em Lotes de Sementes de Schinus molle L.
}

\author{
Pricila Delazeri ${ }^{1}$, Juliana Garlet ${ }^{2}$, Gabriela Fernanda Souza ${ }^{1}$
}

${ }^{1}$ Curso de Engenharia Florestal, Universidade do Oeste de Santa Catarina - UNOESC, Xanxerê/SC, Brasil ${ }^{2}$ Curso de Engenharia Florestal, Universidade do Estado de Mato Grosso - UNEMAT, Alta Floresta/MT, Brasil

\begin{abstract}
RESUMO
Este experimento objetivou avaliar a eficiência do teste de condutividade elétrica para determinar a qualidade fisiológica de lotes de sementes de Schinus molle. No estudo foram utilizados três lotes de sementes armazenados por diferentes períodos (1, 8 e 12 meses), avaliando-se duas quantidades de sementes (50 e 100) e três volumes de água $(25,50$ e $75 \mathrm{~mL})$ para obtenção da solução de embebição e posterior leitura da condutividade elétrica. Foi realizado também o teste de germinação para melhor interpretação dos resultados. Observou-se que os três volumes de água permitiram diferenciar os três lotes para as duas quantidades de sementes utilizadas. O lote com menor período de armazenamento apresentou os menores valores de condutividade elétrica, apresentando melhor qualidade fisiológica e confirmando os resultados do teste de germinação. O teste de condutividade elétrica mostrou-se eficiente para a diferenciação de lotes de sementes de Schinus molle, podendo ser conduzido a $25^{\circ} \mathrm{C}$, com o uso de 50 sementes embebidas em $25 \mathrm{~mL}$ de água por 24 horas.
\end{abstract}

Palavras-chave: aroeira, testes de vigor, sementes florestais.

\section{Electrical Conductivity Test in Batches of Schinus molle L. Seeds}

\begin{abstract}
The objective of this study was to evaluate the efficiency of the electrical conductivity test to determine the physiological characteristics of lots of Schinus molle seeds. In the test, we used three lots of seeds stored for different periods of time, and evaluated two quantities of seeds and three volumes of water to obtain the soaking solution for subsequent reading of electrical conductivity. For conductivity, three volumes of water showed evidence of differences between the three lots for the two quantities of seeds. The lot with shorter storage period presented the lowest electrical conductivity and better physiological quality, confirming the results of the germination test. Thus the electrical conductivity test is efficient in differentiating batches of Schinus molle seeds, and it can be conducted at $25^{\circ} \mathrm{C}$, using 50 seeds soaked in $25 \mathrm{~mL}$ of water for 24 hours.
\end{abstract}

Keywords: aroeira, vigor tests, forest seeds. 
A aroeira salsa (Schinus molle L.) pertence à família Anacardiaceae e no Brasil ocorre do estado de Minas Gerais até o Rio Grande do Sul, sendo uma espécie pioneira, perenofólia e heliófila, que apresenta capacidade de regeneração natural. A espécie vem sendo utilizada em sistemas silvipastoris, para madeira serrada, energia, celulose e papel, material tintorial, óleo resina, alimentação animal, apícola, paisagismo e recuperação ambiental (Carvalho, 2003).

Schinus molle não apresenta dormência em suas sementes e tem potencial germinativo entre 30\% e $80 \%$ (Carvalho, 2003). De acordo com Wielewicki et al. (2006), as sementes de aroeira salsa têm comportamento ortodoxo, podendo ser armazenadas por até 6 meses, sem perda significativa do potencial de germinação. No entanto, a espécie é pouco estudada, tornando-se importante o desenvolvimento de trabalhos a respeito da produção de mudas, bem como das características de suas sementes.

O teste de germinação é o principal mecanismo utilizado para verificar a qualidade de sementes florestais. Adicionalmente, testes de vigor são ferramentas importantes para determinar a qualidade fisiológica de sementes florestais, sendo aprimorados constantemente. Entre eles podem ser citados os testes de envelhecimento acelerado, condutividade elétrica, lixiviação de potássio e tetrazólio (Vazquez et al., 2011). O teste da condutividade elétrica é considerado um dos mais importantes por apresentar maior rapidez, facilidade de execução e possibilidade de ser padronizado como teste de rotina devido à alta reprodutibilidade e eficiência para separação de lotes de sementes quanto ao seu vigor (Vieira \& Carvalho, 1994; Oliveira, 2012; Santos \& Paula, 2005; Lemes et al., 2015).

O teste de condutividade elétrica consiste na imersão das amostras de sementes, já pesadas, em água deionizada, por períodos predeterminados a temperaturas que variam de $25^{\circ} \mathrm{C}$ a $35^{\circ} \mathrm{C}$ (AOSA, 1983). Durante o processo de embebição, ocorre a liberação de solutos nessa água, conforme seu grau de deterioração, resultando assim em altos valores de condutividade elétrica (AOSA, 1983; Marchi \& Cicero, 2002; Oliveira, 2012).

Os solutos exsudados possuem carga elétrica, a qual pode ser detectada por aparelhos chamados condutivímetros, utilizados para a avaliação da qualidade fisiológica das sementes (Marcos, 1990; Oliveira, 2012). Os menores valores representam menor liberação de exsudados, o que indica maior vigor das sementes, apresentando menor intensidade de desorganização nos sistemas de membranas das células (Oliveira, 2012). Barbosa et al. (2012) destacam que o período de embebição e o teor de água das sementes são os principais fatores que influenciam nos resultados dos testes de condutividade elétrica, por isso a necessidade de estudos, para padronização desses fatores, em espécies florestais.

Considerando a carência de estudos de testes de vigor para espécies florestais com metodologias padronizadas, principalmente para aroeira sala, este estudo teve como objetivo avaliar a eficiência do teste de condutividade elétrica para determinar a qualidade fisiológica de lotes de sementes de Schinus molle.

Os lotes de sementes de aroeira salsa utilizados neste estudo provieram de matrizes da área urbana do município de Xanxerê, SC. Após a coleta, as sementes foram transferidas para laboratório, onde permaneceram armazenadas em saco de papel em refrigerador, à temperatura de $5{ }^{\circ} \mathrm{C}$, até a realização dos testes. Os tratamentos utilizados neste experimento foram denominados lotes 1,2 e 3 , sendo o lote 1 composto por sementes armazenadas por 30 dias, o lote 2 por sementes armazenadas por 8 meses e o lote 3 por sementes armazenadas por 12 meses.

A fim de confirmar os resultados do teste de condutividade elétrica, realizou-se o teste de germinação. Esse foi conduzido em delineamento inteiramente casualizado, com quatro repetições de 25 sementes por tratamento (lotes). As sementes foram distribuídas em caixas gerbox, com papel filtro, permanecendo em sala de germinação à temperatura de $25^{\circ} \mathrm{C}$ até formação de plântulas. Foram consideradas germinadas as sementes que deram origem a plântulas normais.

As contagens foram realizadas diariamente até a estabilização da germinação, que ocorreu aos 20 dias. As variáveis avaliadas foram porcentagem de germinação, Índice de Velocidade de Germinação, Peso fresco (g), Comprimento da raiz $(\mathrm{cm})$, e Comprimento da parte aérea $(\mathrm{cm})$ das plântulas.

No teste de condutividade elétrica foram utilizados os três lotes, avaliando-se duas quantidades de sementes: 50 e 100, e três volumes de água: 25, 50 e 75 mL. Assim, 
o experimento foi fatorial com análise de três fatores: 3 lotes X 2 quantidades de sementes (obtidas através da retirada de amostras simples dos lotes para formação da amostra composta) X 3 quantidades de água deionizada. A fim de obter a solução de embebição para posterior leitura de condutividade elétrica, utilizaram-se quatro repetições para cada combinação dos fatores, sendo as sementes mantidas em recipientes de vidro por 24 horas em $\mathrm{BOD}$, à temperatura de $25^{\circ} \mathrm{C}$. O tempo de embebição foi definido com base em trabalhos realizados com outras espécies florestais.

A leitura da solução de embebição foi efetuada utilizando-se um condutivímetro (Digimed DM-3) e o resultado da leitura foi dividido pelo peso da subamostra, expresso em $\mu \mathrm{S} \mathrm{cm}^{-1} \mathrm{~g}^{-1}$ de semente. O delineamento experimental foi inteiramente casualizado e os dados de condutividade elétrica foram analisados separadamente, para cada combinação entre quantidade de sementes e volume de água. Utilizou-se o teste de Bartlett para testar a homogeneidade dos dados, e o de Shapiro Wilk para a normalidade. Os dados que não apresentaram normalidade foram transformados em Log (X). Os dados transformados foram utilizados somente na análise estatística, nas tabelas foram mantidos os dados originais. Posteriormente, os resultados foram submetidos ao teste $\mathrm{F}$ e, pelo teste de Tukey $(\mathrm{p}>5)$, suas médias foram comparadas. Utilizou-se o programa estatístico Assistat 7.6 Beta em todas as análises estatísticas efetuadas neste estudo.

Os parâmetros de germinação e morfológicos avaliados nas sementes dos três lotes de Schinus molle são apresentados na Tabela 1.

Conforme os dados da Tabela 1 , nota-se significativa diferença na germinação dos lotes avaliados, sendo que o lote 1 apresentou elevada taxa de germinação final quando comparado aos demais, evidenciando que o período de armazenamento dos lotes pode ter influenciado nos resultados obtidos, tanto no teste de germinação como nos demais parâmetros morfológicos. Carvalho (2003) e Lorenzi (2000) destacam que sementes de $S$. molle não devem ser armazenadas por período superior a 12 meses.

Avaliando-se os parâmetros morfológicos das plântulas (Tabela 1), constata-se que, para o comprimento de raiz, as plântulas originárias dos lotes 1 e 2 não diferiram, apresentando apenas diferença em relação ao lote 3. Para comprimento da parte área e peso, o lote 1 foi superior, apresentando diferença significativa em relação aos demais, e o mesmo padrão foi observado pra o IVG.

Na Tabela 2 são apresentados os valores de condutividade elétrica obtidos para os lotes de sementes de Schinus molle associados a diferentes quantidades de sementes e de água deionizada para embebição.

Analisando-se a Tabela 2 é possível verificar que quando se utilizaram 50 sementes com os três volumes de água (25, 50 e $75 \mathrm{~mL}$ ), foi possível separar os lotes de sementes. Observa-se o mesmo padrão quando se utilizaram 100 sementes associadas a diferentes quantidades de água deionizada, e houve diminuição do valor da condutividade com o aumento do volume de água. Resultados semelhantes foram observados por Dalanhol et al. (2014) em sementes de Bowdichia virgilioides Kunth, por Ataíde et al. (2012) em Pterogyne nitens, Araújo et al. (2011) em Jatropha curcas L. (Pinhão manso) e por Torres et al. (2015) em Coriandrum sativum L. (coentro), ou seja o aumento do volume de água diminuiu o valor da condutividade elétrica.

Pereira \& Martins (2012) relatam a importância de estudos com o volume de água para embebição em testes de condutividade elétrica para análise de sementes, buscando assim uma padronização de metodologias,

Tabela 1. Dados de germinação e parâmetros morfológicos de plântulas de Schinus molle.

Table 1. Data germination and morphological parameters of Schinus molle seedlings.

\begin{tabular}{cccccc} 
Lotes & GF $\%$ & $\begin{array}{c}\text { Comprimento de } \\
\text { raiz }(\mathbf{c m})\end{array}$ & $\begin{array}{c}\text { Comprimento da parte } \\
\text { aérea }(\mathbf{c m})\end{array}$ & Peso fresco $(\mathbf{g})$ & IVG \\
\hline 1 & $71,00 \mathrm{a}^{*}$ & $2,71 \mathrm{a}$ & $3,65 \mathrm{a}$ & $0,62 \mathrm{a}$ & $8,03 \mathrm{a}$ \\
\hline 2 & $11,00 \mathrm{~b}$ & $2,28 \mathrm{a}$ & $1,90 \mathrm{~b}$ & $0,11 \mathrm{~b}$ & $1,20 \mathrm{~b}$ \\
\hline 3 & $8,00 \mathrm{~b}$ & $1,00 \mathrm{~b}$ & $0,82 \mathrm{c}$ & $0,10 \mathrm{~b}$ & $0,70 \mathrm{~b}$ \\
\hline
\end{tabular}

GF = Germinação Final (20 dias após instalação do teste); IVG = índice de velocidade de germinação; ${ }^{*}$ médias seguidas pela mesma letra na coluna não diferem estatisticamente pelo teste de Tukey $(\mathrm{p}>0,5)$. 
Tabela 2. Condutividade elétrica $\left(\mu \mathrm{S} \mathrm{cm}^{-1} \mathrm{~g}^{-1}\right)$ de sementes de Schinus molle em função dos lotes, do número de sementes e do volume de água deionizada.

Table 2. Electrical conductivity $\left(\mu \mathrm{S} \mathrm{cm} \mathrm{cm}^{-1}\right)$ in Schinus molle seeds, for different batches and different quantities of seeds and deionized water.

\begin{tabular}{|c|c|c|c|}
\hline \multirow{2}{*}{ Lotes } & \multicolumn{3}{|c|}{50 sementes } \\
\hline & $25 \mathrm{~mL}$ & $50 \mathrm{~mL}$ & $75 \mathrm{~mL}$ \\
\hline 1 & $290,00 \mathrm{aC}$ & $166,93 \mathrm{bC}$ & $112,18 \mathrm{cC}$ \\
\hline 2 & $453,43 \mathrm{aB}$ & $268,21 \mathrm{bB}$ & $215,81 \mathrm{bB}$ \\
\hline 3 & $921,87 \mathrm{aA}$ & $457,50 \mathrm{bA}$ & $342,81 \mathrm{cA}$ \\
\hline \multirow{2}{*}{ Lotes } & \multicolumn{3}{|c|}{100 sementes } \\
\hline & $25 \mathrm{~mL}$ & $50 \mathrm{~mL}$ & $75 \mathrm{~mL}$ \\
\hline 1 & $258,90 \mathrm{aC}$ & $131,25 \mathrm{bC}$ & $87,00 \mathrm{cC}$ \\
\hline 2 & $458,90 \mathrm{aB}$ & $263,75 \mathrm{bB}$ & $159,96 \mathrm{cB}$ \\
\hline 3 & $732,65 \mathrm{aA}$ & $462,50 \mathrm{bA}$ & $292,96 \mathrm{cA}$ \\
\hline
\end{tabular}

Médias seguidas pela mesma letra maiúscula na coluna e minúscula na linha não diferem estatisticamente pelo teste de Tukey ( $\mathrm{p}>0,5)$; obs: dados não transformados.

pois a utilização de volumes diferentes de água, para a mesma espécie, impossibilita a comparação dos resultados.

Os resultados da Tabela 2 corroboram os dados observados na Tabela 1, na qual se verifica que o lote 1 apresentou o maior percentual de germinação e, na avaliação da condutividade, os menores valores, quanto maior o valor da condutividade, menor é o vigor das sementes. Essa maior quantidade de lixiviados no exsudato ocorre devido à perda da integridade das membranas celulares (Binotti et al., 2008). Conforme a semente vai envelhecendo, acontece a sua deterioração, consequentemente, a perda da integridade dos sistemas de membranas da célula, ocasionando aumento de sua permeabilidade, o que resulta na lixiviação de eletrólitos e maior condutividade elétrica (Santos \& Paula, 2005; Oliveira, 2012).

O teste de condutividade elétrica mostrou-se eficiente para a diferenciação de lotes de sementes de Schinus molle, podendo ser conduzido a $25^{\circ} \mathrm{C}$, com o uso de 50 sementes embebidas em $25 \mathrm{~mL}$ de água por 24 horas.

\section{STATUS DA SUBMISSÃO}

Recebido: 15 abr., 2015

Aceito: 21 fev., 2016

\section{AUTOR(ES) PARA CORRESPONDÊNCIA}

\section{Juliana Garlet}

Departamento de Engenharia Florestal, Faculdade de Ciências Biológicas e Agrárias, Universidade do Estado de Mato Grosso UNEMAT, Campus II, Av. Perimetral Rogério Silva, s/n, Jardim Flamboyant, CEP 78580-000, Alta Floresta, MT, Brasil e-mail: julianagarlet@unemat.br

\section{REFERÊNCIAS}

Araújo RF, Zonta JB, Araujo EF, Donzeles SML, Costa GM. Teste de condutividade elétrica para sementes de pinhão-manso (Jatropha curcas L.). Idesia 2011; 29(2): 7986. http://dx.doi.org/10.4067/S0718-34292011000200010.

Association of Official Seed Analysis - AOSA. Seed vigour handbook. In: AOSA. The handbook of seed testing. East Lansing: AOSA; 1983

Ataíde GM, Flôres AV, Borges EEL, Resende RT. Adequação da metodologia do teste de condutividade elétrica para sementes de Pterogyne nitens Tull. Revista Brasileira de Ciências Agrárias 2012; 4(7): 635-640. http://dx.doi. org/10.5039/agraria.v7i4a1688.

Barbosa RM, Silva CB, Medeiros MA, Centurion MAPC, Vieira RD. Condutividade elétrica em função do teor de água inicial de sementes de amendoim. Ciência Rural 2012; 42(1): 45-51. http://dx.doi.org/10.1590/S010384782012000100008 .

Binotti FFS, Haga KI, Cardoso ED, Alves CZ, Sá ME, Arf, O. Efeito do período de envelhecimento acelerado no teste de condutividade elétrica e na qualidade fisiológica de sementes de feijão. Acta Scientiarum. Agronomy 2008; 2(30): 247-254.

Carvalho PER. Espécies arbóreas brasileiras. Brasília: Embrapa; 2003.

Dalanhol SJ, Rezende EH, Abreu DCA, Nogueira AC. Teste de condutividade elétrica em sementes de Bowdichia virgilioides Kunth. Floram 2014; 21(1): 69-77. http://dx.doi. org/10.4322/floram.2014.013.

Lemes ES, Oliveira S, Rodrigues RR, Almeida AS, Meneghello GE, Tunes LM. Avaliação do potencial fisiológico de lotes de aveia preta por meio do teste de condutividade elétrica. Tecnologia \& Ciência Agropecuária 2015; 9(2): 5-10.

Lorenzi H. Árvores brasileiras: manual de identificação e cultivo de plantas arbóreas do Brasil. 2. ed. São Paulo: Plantarum; 2000.

Marchi JL, Cicero SM. Procedimentos para a conduçãodo teste de condutividade elétrica em sementes. Informativo ABRATES 2002; 1(12): 2-3. 
Marcos J. Fo. Estudo comparativo de métodos para avaliação da qualidade fisiológica de sementes de soja, com ênfase ao teste de condutividade elétrica. Pesquisa Agropecuaria Brasileira 1990; 12(25): 1805-1815.

Oliveira S. Tecnologia de sementes florestais: espécies nativas. Curitiba: UFPR; 2012.

Pereira MD, Martins S Fo. Adequação da metodologia do teste de condutividade elétrica para sementes de cubiu (Solanum sessiliflorum DUNAL). Revista Agrarian 2012; 5(16): 93-98.

Santos SRG, Paula RC. Teste de condutividade elétrica para avaliação da qualidade fisiológica de sementes Sebastiania commersoniana (Bail) Smith \& Downs Euphorbiaceae. Revista brasileira de Sementes 2005; 2(27): 136-145.

Torres SB, Paiva EP, Almeida JPN, Benedito CP, Carvalho SMC. Teste de condutividade elétrica na avaliação da qualidade fisiológica de sementes de coentro. Revista Ciência Agronômica 2015; 46(3): 622-629.

Vazquez GH, Bertolin DC, Spegiorin CN. Testes de envelhecimento acelerado e de condutividade elétrica para avaliar a qualidade fisiológica de sementes de sorgo (Sorghum bicolor (L.) Moench). Revista Brasileira de Biociências 2011; 9(1): 18-24.

Vieira RD, Carvalho NM, editores. Testes de vigor em sementes. Jaboticabal: FUNEP; 1994.

Wielewicki AP, Leonhardt C, Schlindwein G, Medeiros ACS. Proposta de padrões de germinação e teor de água para sementes de algumas espécies florestais presentes na região sul do Brasil. Revista Brasileira de Sementes 2006; 28(3): 191-197. http://dx.doi.org/10.1590/S010131222006000300027 . 